\title{
Evaluation of Echocardiography as a Marker of Cardiovascular Risk in Obese Children and Adolescents
}

\author{
Simone Helena Caixe ${ }^{\mathrm{a}, \mathrm{e}}$, Augusto Cesar Garcia Saab Benedeti ${ }^{\mathrm{a}}$, Jorge Garcia ${ }^{\mathrm{a}}$, \\ Wellington de Paula Martins ${ }^{\mathrm{b}}$, Francisco Mauad Filho ${ }^{\mathrm{b}}$, \\ Luiz Antonio Del Ciampo ${ }^{\mathrm{c}}$, Carlos Alberto Nogueira-de-Almeida ${ }^{\mathrm{d}}$
}

\begin{abstract}
Background: Childhood obesity is a global epidemic and its prevalence has increased from the early 1970s to the 1990s. The objective of the study was to compare the echocardiographic parameters of left ventricular (LV) geometry between obese and non-obese children.
\end{abstract}

Methods: A prospective cross-sectional study with 71 individuals aged 7 - 11 years, from Ribeirao Preto metropolitan region, was conducted. Personal data (sex and date of birth) were obtained for each subject, as well as anthropometric measurements (weight, height and abdominal circumference), arterial pressure and an echocardiogram. The individuals were classified according to their nutritional status in "obese" and "non-obese" groups.

Results: There was no difference in gender $(\mathrm{P}=0.81)$, with $48.6 \%$ boys in obese group and $52.9 \%$ in non-obese group. Similarly, there was no difference in age $(\mathrm{P}=0.72)$, with a mean of $106.97 \pm 19.59$ months in obese group and a mean of $105.50 \pm 13.61$ months in non-obese group. Systolic and diastolic pressure values were higher in obese group. The study showed LV mass, LV 3 index, systolic arterial pressure and diastolic arterial pressure when the two groups were compared. LV 1 index, LV 2 index and relative LV wall thickness did not differ between groups.

Manuscript accepted for publication August 28, 2014

${ }^{a}$ Faculty of Health Technology, University of Ribeirao Preto, Rua Cerqueira Cesar 660, Ribeirao Preto, SP, CEP 14020060, Brazil

${ }^{b}$ Faculty of Health Technology and Faculty of Medicine of Ribeirao Preto, University of Sao Paulo, Rua Cerqueira Cesar 660, Ribeirao Preto, SP, CEP 14020060, Brazil

${ }^{\mathrm{c}}$ Department of Puericulture and Pediatrics, Faculty of Medicine of Ribeirao Preto, University of Sao Paulo, Avenida Bandeirantes 3900, Ribeirao Preto, SP, CEP 14049900, Brazil

${ }^{\mathrm{d}}$ University of Ribeirao Preto, Avenida Costabile Romano 2201, Ribeirao Preto, SP, CEP 14096900, Brazil

${ }^{\mathrm{e}}$ Corresponding Author: Simone Helena Caixe, University of Ribeirao Preto, Rua Cerqueira Cesar 660, Ribeirao Preto, SP, CEP 14020060 ,

Brazil.Email: simone@ultra-sonografia.com.br
Conclusion: The study contributes to demonstrate the importance of transthoracic echocardiography as a marker of cardiovascular risk in obese children and adolescents.

Keywords: Obesity; Child obesity; Echocardiography; Left ventricular hypertrophy

\section{Introduction}

Childhood obesity is a global epidemic. Over the last decades, its prevalence has increased from the early 1970s to the end of the 1990s [1]. In Brazil, according to the 2011 2022 plan of strategic actions for coping with chronic diseases of the Ministry of Health, the prevalence of excess weight among children aged 5 - 9 years has reached $33.5 \%$ and the prevalence of obesity has reached $14.3 \%$ [2]. Excess weight has been diagnosed in $20 \%$ of children and adolescents aged $10-19$ years, and obesity has been diagnosed in $9.9 \%$ with a $4 \%$ rate among girls and $5.9 \%$ rate among boys [2].

Studies conducted in the 1990 decade already showed risk factors for cardiovascular diseases and evidence that atherosclerosis starts early among obese children and adolescents [3]. A study published in the city of Belo Horizonte, Brazil, has demonstrated that overweight or obese students had a 3.6 times higher risk to develop high systolic arterial pressure and a 2.7 higher risk to develop high diastolic arterial pressure compared to normal-weight students [4], with a combination of risk factors for the development of metabolic syndrome being identified. One of each five participants $(19.3 \%)$ presented four risk factors: elevated total cholesterol $(>200 \mathrm{mg} / \mathrm{dL})$, body mass index $(\mathrm{BMI})>85$ th percentile, systolic arterial pressure $>90$ th percentile, and diastolic arterial pressure $>90$ th percentile. The participants were considered children and adolescents. The study also showed that students with low levels of physical activity or with a sedentary life style had higher arterial pressure levels and a lipid profile indicative of increased risk to develop atherosclerosis.

Thus, in view of the fact that obesity may course with 
cardiovascular changes such as concentric left ventricular (LV) hypertrophy in obese children with arterial hypertension and concentric remodeling in normotensive children, as well as the fact that hypertension and concentric LV hypertrophy are independent predictors of diastolic LV function, there is an evident need for early detection of these changes [5].

Dhuper et al [6] studied 213 children and adolescents with BMI $>95$ and 130 normal-weight ones with $\mathrm{BMI}<85$ and no structural disease, and investigated the association of obesity with hypertension by echocardiography. The authors detected a more prevalent geometric pattern of concentric LV remodeling in obese subjects, and a more prevalent pattern of concentric hypertrophy in obese subjects with hypertension. Obesity, hypertension and concentric hypertrophy were independent predictors of diastolic dysfunction. The authors pointed out that various changes in cardiac function and morphology can be seen early in life.

In view of the fact that echocardiography is an important diagnostic method for the assessment of changes in cardiac structure and function and the epidemic of childhood obesity, the objective of the present study was to assess the present or not of early changes in LV structure in obese children and adolescents.

\section{Subjects and Methods}

A cross-sectional study was conducted in 71 individuals from the population of Ribeirao Preto city and the metropolitan region seen at basic health units and examined at the Faculty of Health Technology (FATESA/EURP) located in Ribeirao Preto, State of Sao Paulo, Brazil.

The health units of the city of Ribeirao Preto and its metropolitan region were informed about the project by ample dissemination and the managers of the units were invited to refer volunteer children who fulfilled the following inclusion criteria: age between 7 years completed and 11 years uncompleted and consent form signed by their parents or persons legally responsible. This age group was chosen to minimize the hormonal influence that exists in adolescence [7]. Exclusion criteria were presence of diseases that might interfere with growth or anthropometry, presence of congenital and acquired heart diseases, medications that might influence the cardiovascular system, and BMI z-score $\leq 2$. The participants in the study were evaluated by a semiologic approach and by an echocardiographic exam performed by a single examiner. Personal data (sex and date of birth) were obtained from each participant and anthropometric measurements (weight, height and abdominal circumference) were carried out; arterial pressure measure and echocardiogram were performed once time. The anthropometric, arterial pressure and echocardiographic measurements were obtained in a blind manner, i.e. the examiner did not know if she was a child do- ing exams in the control group or the study group, since the anthropometric data were always evaluated after the completion of the examinations and after data collection and tabulation, the children were stratified into two groups according to nutritional status, i.e., non-obese (BMI z-score between -2 and +2 ) and obese (BMI z-score higher than +2 and abdominal circumference above the 90th percentile in the Freedman table [8]. A medical report was prepared and made available to the person legally responsible for the child. An initial statistical analysis was performed in order to determine whether the groups were homogeneous in age and sex distribution.

A calibrated Krato-Cas electronic scale, Portable Light Line model, Brazil (maximum load of $150 \mathrm{~kg}$ and precision of $50 \mathrm{~g}$ ) was used to measure weight and a portable Kratos-Cas anodized aluminum anthropometer with a $2 \mathrm{~m}$ ruler and $1 \mathrm{~mm}$ divisions and a fixed marker was used to measure height. An inextensible metric tape with $0.1 \mathrm{~cm}$ precision was used to measure the waist circumference at the natural waistline, i.e., the midpoint between the anterosuperior iliac crest and the last rib. Systolic arterial pressure was measured with a Tycos, an android device with a rubber bag whose dimensions followed the different arm circumference of children listed in the table of the VI Brazilian Directives of Arterial Pressure [9].

The parameters analyzed by the echocardiogram were LV mass and relative LV wall thickness. The LV mass indices were obtained with three types of evaluation (LV mass/ body surface, LV mass/height ${ }^{2}$ and LV mass/height ${ }^{2.7}$ ).

The values of all echocardiographic parameters were obtained by calculating the mean of three consecutive cycles. LV mass was calculated using the $\mathrm{M}$ mode and the formula of Devereux [7], as recommended by the American Society of Echocardiography (ASE) [7, 10].

The LV mass indices were calculated in three different ways: for the LV 1 index, the LV mass was divided by the body surface (BSA) (expressed in meters) $)^{2}\left(\mathrm{~g} / \mathrm{m}^{2}\right)$ (formula of Haycock [11]); for the LV 2 index, the LV mass was divided by height (expressed in meters) ${ }^{2.7}\left(\mathrm{~g} / \mathrm{m}^{2.7}\right)$, and for the LV 3 index, the LV mass was divided by height (expressed in meters $)^{2}\left(\mathrm{~g} / \mathrm{m}^{2}\right)$. According to Lang et al [7], the use of left LV mass in children is hampered by the need to index the measurements in relation to the body size of the patient. Relative wall thickness (RWT) was calculated by multiplying the thickness of the posterior LV wall by 2 and dividing it by the diastolic LV diameter, as recommended by ASE and by the European Society of Echocardiography [7]. The posterior LV wall and the diastolic LV diameters were measured as described earlier.

The individuals were stratified as non-obese and obese on the basis of their anthropometric data and of the distribution of the echocardiographic parameters, as well as the abdominal circumference and arterial pressure. The data were compared between groups by comparing the means and the $\mathrm{P}$ values were calculated by the unpaired $t$-test. Gender dis- 
tribution was tested by the Fisher exact test.

The study was approved by the Ethics Committee for Research on Human Beings of University of Ribeirao Preto (protocol no. 215.788) and the parents or persons legally responsible for the children gave written informed consent to participate.

\section{Results}

Table 1 presents the results of the variables studied. All individuals with a BMI z-score greater than +2 had an abdominal circumference above the 90th percentile of the Freedman table [8]. There was no difference in gender distribution between groups ( $\mathrm{P}=0.81$ ), with $48.6 \%$ of males being observed in the "obese" group and $52.9 \%$ in the "non-obese" group. Also, there was no difference in age $(\mathrm{P}=0.72)$, with a mean of $106.97 \pm 19.59$ months in the "obese" children group and a mean of $105.50 \pm 13.61$ months in the "nonobese" children group.

Systolic and diastolic pressure values were higher in the group of obese children. The study showed a significant difference for LV mass, LV 3 index, systolic arterial pressure and diastolic arterial pressure when the two groups were compared. LV 1 index, LV 2 index and relative LV wall thickness did not differ between groups.

\section{Discussion}

In this study significant differences in waist circumference, LV mass, LV 3 index, systolic blood pressure and diastolic blood pressure were found between the obese group and the group of non-obese children.

According to Daniels et al [12], LV hypertrophy was established as an independent for the development of cardiovascular morbidity and mortality risk factor, and therefore understanding the determinants of LV mass in children and adolescents would be important. They comment that LV mass increases during childhood and adolescence to body growth but suggests that, among other factors, blood pressure also affects the LV mass. Also, they refer that lean body mass also increases in childhood obesity and that the mechanism of this increase associated with obesity is poorly understood, but should be responsible for some pathological effects of obesity, such as hypertension or LV hypertrophy. Further, they suggest that blood pressure is important in determining LV mass and has the additional effect of obesity and conclude that longitudinal studies are needed to better understand these mechanisms.

In 1998 it was reported that the ability to measure LV mass by echocardiography played a relevant role regarding the pathogenesis of cardiovascular disease. This fact acquired even greater importance according to the conclusions 
of the Framingham Study, which indicated that an increase in LV mass represented a risk factor for cardiovascular morbidity independently of conventional risk factors including obesity and hypertension [13].

A literature search using the keywords "left ventricle", "left-ventricular hypertrophy", "cardiac hypertrophy", "obesity", "hypertension" and "echocardiography" was performed and 22 studies including 5,486 obese individuals were considered and the prevalence of LV hypertrophy was $56 \%$ and data provided by 15 studies, with 4,999 obese individuals, and 6,623 non-obese controls showed that probability of having LV hypertrophy was much higher in obese individuals and among obese LV hypertrophy, eccentric hypertrophy was more frequent that the concentric. The authors concluded that LV hypertrophy is present in a consistent fraction of the obese individuals and that eccentric hypertrophy prevails over the concentric. They related that obesityrelated LV hypertrophy results in LV dysfunction. Thus, the prevention and treatment of obesity may contribute to reduce morbidity, because the weight loss is associated with reduction of LV hypertrophy in obese individuals [14].

Kinik et al [15] investigated 30 obese children by a standard echocardiogram and by tissue Doppler and compared them to a control group. Blood glucose, lipid and insulin levels were determined in the obese children. Systolic and diastolic LV function was normal. The mass index (in which mass was indexed according to height ${ }^{2.7}$ ), the RWT and the final diastolic diameter of the LV were significantly greater in the obese children. The authors concluded that LV hypertrophy was present in obese children, as demonstrated by the increased LV mass, and that insulin, low density lipoprotein (LDL) and triglyceride levels were associated with LV hypertrophy. Tissue Doppler of the right ventricle and of the interventricular septum revealed subclinical variations in diastolic function.

Other investigators consider obesity to be a risk factor for hypertension and LV hypertrophy, but the association between obesity, hypertension and LV hypertrophy has not been extensively studied in adolescents. According to these authors, obese adolescents have a significantly higher prevalence of hypertension and of LV hypertrophy and studies have suggested a direct negative effect of obesity on cardiovascular function starting before the age of adolescence [16].

Senatorova et al [17] investigated 141 adolescents, and 114 of them obese and 27 overweight, using an echocardiogram with tissue Doppler in order to assess LV geometry and systolic and diastolic LV function. The authors established that, in adolescents, the process of cardiac remodeling starts with initial thickening of the interventricular septum and that obesity is associated with eccentric myocardial hypertrophy. Cardiac output increased significantly as the result of increased final LV diastolic volume without causing significant variations in intracardiac kinetics, a fact that contributed to the preservation of ejection fraction within normal reference values.

Daniels et al [12] have proposed an allometric definition of excess mass $\left(>51 \mathrm{~g} / \mathrm{m}^{2}\right)$ and of hypertrophy patterns (concentric, eccentric, and concentric remodeling) for a cohort of children under follow-up with essential arterial hypertension. In these children distribution of LV mass instead of excessive hypertrophy was obtained, with a small but significant percentage of LV mass at pathological levels and a significant percentage of a concentric hypertrophy pattern associated with increased mortality in adults. These findings were clinically significant, establishing the concept of the existence of a target organ of hypertension in children, so that the monitoring of LV mass would be important for the management of hypertension in childhood.

In the present study, the values detected for LV mass were significantly higher for obese children $(79.49 \pm 21.21$, $\mathrm{P}<0.01)$ than for non-obese children $(56.74 \pm 11.77)$. However, it should be kept in mind that this result may have a lower clinical meaning for obesity since it was not indexed according to height, i.e., it was not corrected as suggested above by Daniels et al [12].

The relations between LV mass and height or body surface are not linear, but are regulated by allometric (growth) functions which are exponential [18]. Indexing permits to consider normal lean body mass growth in childhood. However, the pathological effects in overweight or obese individuals should not be overlooked, with the measurement of indexed LV mass at the beginning of childhood being directly comparable to a subsequent measurement during adolescence and maturity [7].

Lang et al [7] refer to dividing LV mass by height (meters) increased to a power of 2.7 , in older children and adolescents is generally used. Although, in younger children (8 years old), the LV mass by height (meters) increased to a power of 2.0 appears to be the most appropriate.

In a study on 544 essential hypertensive, 173 men and 371 women ages ringing from 13 to 84 years and 17 to 80 years respectively, and 106 normotensive individuals, Rosa et al [19], using an M mode echocardiogram and LV mass indexed according to body surface (LV mass/body surface area) and to height squared (LV mass $/$ height $^{2}$ ), compared the two LV mass indices in the two populations stratified according to BMI. However, indexing by body surface area did not show significant differences between subgroups, whereas indexing by height squared showed significantly greater differences according to the increase in BMI in the hypertensive and normotensive populations. The authors concluded that LV hypertrophy calculated by LV/body surface area has been underestimated in obese individuals, since this type of indexing considers obesity to be a physiological variable. In contrast, indexing by height squared reveals the difference existing in subgroups with different BMI and therefore is more appropriate for the determination of LV hypertrophy in obese populations. 
Hashimoto et al [20], in a study conducted in order to determine the best method for the standardization of LV mass and to assess LV hypertrophy induced by obesity, observed that the application of height ${ }^{1.85}$ to men and height ${ }^{1.72}$ to women is an appropriate method for the indexing of LV mass in children and adolescents. Di Bonito et al [21] studied the influence of central obesity on LV mass and on LV function in obese children. They calculated LV mass according to the Penn convention and indexed according to height ${ }^{2.7}$ and detected an increased left ventricular mass index (LVMI) and preserved LV function in obese children, concluding that central adiposity is the major determinant of LV mass.

A study compared the variations in LV mass index and LV mass between two generations of healthy children and adolescents aged 2 - 19 years who were submitted to an echocardiogram. BMI and LV mass index were significantly higher in the 2008 generation than in the generation from 1986 to 1989 . Thus, it was concluded that the children of the younger generation had higher BMI and LV mass index values and higher predictive factors of cardiovascular risk than the children of the previous generation [22].

Bostanci et al [23] investigated the relation between metabolic syndrome and LV mass index and concluded that LV hypertrophy commonly occurs in the pediatric metabolic syndrome and is associated with systolic hypertension and insulin resistance, and that the LV mass index should be routinely measured in order to predict the cardiovascular risks of these patients.

In the current study, when LV mass was indexed according to body surface calculated by the formula of Haycock [11], there was no difference between obese and normalweight individuals. The same was observed for the LV 2 mass index when LV mass was indexed by height ${ }^{2.7}$. Thus, when the LV 3 mass index was calculated according to height squared, a significant $\mathrm{P}$ value was obtained $(\mathrm{P}<0.01)$, with higher values detected among obese $(38.32 \pm 9.84)$ than non-obese (32.56 \pm 7.16$)$ individuals.

Few studies present reference values for LVMI and RWT in children. In the journal Pediatrics in 2004, a collaborative study of the Pediatric Hypertension Association, a retrospective review with data from 1998 to 2001, from three different locations (University of Texas at Houston, Children's Hospital Medical Center of Cincinnati and Medical College of published Georgia), conducted with 129 patients with a mean age of $13.6 \pm 3.6$ years, with a range between 4.2 and 22 years, in which the LVMI was calculated as LV mass divided the time raised to the power of 2.7. In this study we defined the criteria for LV hypertrophy in children and adults. The criteria for LV hypertrophy in adults was defined as LVMI $>51 \mathrm{~g} / \mathrm{m}$ increased to 2.7 and pediatric criteria for LVH was LVMI $>38.6 \mathrm{~g} / \mathrm{m}$ raised to 2.7 . The RWT was considered abnormal if $\geq 0.41$. LV geometry was classified as concentric, concentric remodeling, eccentric or normal. Thus, concentric when LVMI and RWT were increased; concentric remodeling when LVMI was normal, but the ERP was increased; eccentric, when LVMI was increased, but the ERP was normal; and normal, when LVMI and ERP were normal [24].

In the current study the relative LV wall thickness was not significantly different when comparing the values obtained in groups of non-obese children and adolescents $(30.74 \pm 6.09)$ and obese $(31.27 \pm 5.11)$.

In addition to the echocardiographic parameters, also the systolic and diastolic blood pressure and waist circumference in children and non-obese and in obese children and adolescents were evaluated, and we obtained higher values of systolic and diastolic blood pressure in obese children and adolescents, as well as larger waist circumference.

Paschoal et al [25] conducted a study with 91 students in public schools in the city of Campinas, SP, aged $7-8$ years, when they were evaluated by anthropometric and clinical profile and its relationship with the metabolic syndrome. These children were divided into three groups: normal-weight children suffering from overweight and suffering from obesity. Forty-nine point four percent normal weight, $13.1 \%$ overweight and $37 \%$ obese were obtained. Weight, height, blood pressure and waist and arm circumferences were measured. Yielded lower values of systolic blood pressure in children eutrophic (value of $88.2 \pm 4.0 \mathrm{~mm} \mathrm{Hg}$ ) compared with obese children $(93.4 \pm 7.2 \mathrm{~mm} \mathrm{Hg})$. Abdominal circumferences and brachial were statistically different in the three groups of children, higher in obese children and presented with lower values in eutrophic. It was found that systolic blood pressure and diastolic blood pressure correlated with waist circumference in the three groups of children.

Still, Paschoal et al [25] report that the findings in their study are consistent with epidemiological studies that met three times greater risk of higher blood pressure levels in obese children compared with normal weight. They note that other studies showed a significant elevation of blood pressure in obese children and stated that there is tendency to childhood obesity cause increase in blood pressure, especially the systolic.

According to Iampolsky et al [26] the pathophysiology of changes in blood pressure due to increased body weight in children is not well understood. However, in adults, three related mechanisms are known: insulin resistance and hyperinsulinemia; increased sympathetic nervous system activity and changes in vascular structure and function. However, recently, central adiposity and insulin resistance were also indicated as determinants of elevated blood pressure in children. Also, they note that excess abdominal fat is associated with increased risk of developing chronic diseases, especially cardiovascular. And, they say that abdominal circumference has good relationship with visceral fat.

Finally, Iampolsky et al [26] refer that is very important to include the measurement of blood pressure in the pediatric clinical examination, enabling early intervention, since 
elevated blood pressure in childhood is associated with the persistence of these in adulthood and thus increase the risk for cardiovascular diseases such as hypertension. And, they comment as the development of these diseases in adulthood increases risk as the number of measures changed during childhood and adolescence, there may be need to detect and intervene early.

In another study, De Moraes et al [27] involved 817 children and adolescents aged $6-13$ years enrolled in public schools in the municipality of Vila Velha, Espirito Santo, who were assessed for height, weight and blood pressure. Regarding the general characteristics of the sample, De Moraes et al [27] obtained homogeneity in relation to sex, being represented by $51 \%$ of boys and $49 \%$ girls. The mean age was $8.8 \pm 1.6$ years. The percentage of obese children ranged between $67 \%$ and $73 \%$, and the percentage of people with overweight or obese children, between $21 \%$ and $27 \%$. They comment that there was a strong correlation between the presence of overweight and the occurrence of elevated blood pressure, both systolic and diastolic. And it was demonstrated that, regardless of nutritional status criterion levels $\mathrm{z}$ score increase in blood pressure as low weight moves obesity.

Falkner et al [28] examined the relative effects of arterial pressure and obesity on LV mass among Afro-American adolescents and observed that metabolic and inflammatory risk factors were associated with obesity, but not with LV mass index independently (LV mass/height ${ }^{2.7}$ ). The authors concluded that the prevalence of LV hypertrophy is higher among obese Afro-American adolescents with arterial pressure $\geq 120 / 80 \mathrm{~mm} \mathrm{Hg}$, and that an independent association of LV mass with arterial hypertension starts in the 75th percentile of systolic arterial pressure.

De Almeida et al [29] conducted a study in which 624 individuals of both sexes were evaluated aged $7-18$ years obtained from two public schools, as the blood dosage, fasting insulin, glucose, total cholesterol and leptin and who had also measured the weight, height and waist circumference. This study compared the evaluation of abdominal circumference performed by two tables of references, and showed that despite the reference Taylor et al [30] to be more sensitive to hyperlipidemia, the reference Freedman et al [7] had higher specificity, being more suitable for clinical use, and you can use it instead of blood dosages that often are not available. Thus, given the importance of obesity has been suggested that screening by reference Freedman et al [8] was always performed, emphasizing the measurement of waist circumference as a mandatory review in the examination of the child.

In conclusion, the present cross-sectional study showed that the group of obese children had higher arterial pressure levels and higher abdominal circumference values, in addition to differences in echocardiographic parameters such as LV mass index. Although, we have found a significant dif- ference in LV mass indexed by height squared, as well as LV mass among the obese and non-obese, the breadth of significance is uncertain, since the population was small and we could not stratify by age group. Further, because the mass was indexed by height to the 2.7 power and 2 regardless of the age group indicated as advocated by Lang et al [7].

Considering the present findings and the fact that the persistence of obesity in these healthy children until they reach adolescence can course with arterial hypertension and/ or LV hypertrophy, both considered to be independent risk factors for cardiovascular changes, as is the case for obesity, it can be clearly seen that transthoracic echocardiography is important as a marker of cardiovascular risk in children, especially because it is biologically safe, minimally invasive, and easily accessible.

\section{Grant Support}

The equipment for the exams was provided by the Faculty of Health Technology.

\section{Conflicts of Interest}

Nothing to declare.

\section{References}

1. Han JC, Lawlor DA, Kimm SY. Childhood obesity. Lancet. 2010;375(9727):1737-1748.

2. Ministerio da Saude. Plano de acoes estrategicas para o enfrentamento das doencas cronicas nao transmissiveis 2011-2022 Brasilia: Ministerio da Saude; 2011.

3. Oliveira CLD, Mello MTD, Cintra IDP, Fisberg M. Obesidade e sindrome metabolica na infancia e adolescencia. Rev Nutr. 2004;17:237-245.

4. Ribeiro RQ, Lotufo PA, Lamounier JA, Oliveira RG, Soares JF, Botter DA. [Additional cardiovascular risk factors associated with excess weight in children and adolescents: the Belo Horizonte heart study]. Arq Bras Cardiol. 2006;86(6):408-418.

5. Caixe SH, De Almeida CAN. Ecocardiografia para avaliacao cardiovascular de criancas com sobrepeso/obesidade: uma revisao. Int J Nutr. 2013;6:108-113.

6. Dhuper S, Abdullah RA, Weichbrod L, Mahdi E, Cohen HW. Association of obesity and hypertension with left ventricular geometry and function in children and adolescents. Obesity (Silver Spring). 2011;19(1):128-133.

7. Lang RM, Bierig M, Devereux RB, Flachskampf FA, Foster E, Pellikka PA, Picard MH, et al. Recommendations for chamber quantification: a report from the American Society of Echocardiography's Guidelines 
and Standards Committee and the Chamber Quantification Writing Group, developed in conjunction with the European Association of Echocardiography, a branch of the European Society of Cardiology. J Am Soc Echocardiogr. 2005;18(12):1440-1463.

8. Freedman DS, Dietz WH, Srinivasan SR, Berenson GS. The relation of overweight to cardiovascular risk factors among children and adolescents: the Bogalusa Heart Study. Pediatrics. 1999;103(6 Pt 1):1175-1182.

9. VI Diretrizes Brasileiras de Hipertensao. Arq Bras Cardiol. 2010;95:I-III.

10. Lai WW, Geva T, Shirali GS, Frommelt PC, Humes RA, Brook MM, Pignatelli RH, et al. Guidelines and standards for performance of a pediatric echocardiogram: a report from the Task Force of the Pediatric Council of the American Society of Echocardiography. J Am Soc Echocardiogr. 2006;19(12):1413-1430.

11. Haycock GB, Schwartz GJ, Wisotsky DH. Geometric method for measuring body surface area: a heightweight formula validated in infants, children, and adults. J Pediatr. 1978;93(1):62-66.

12. Daniels SR, Loggie JM, Khoury P, Kimball TR. Left ventricular geometry and severe left ventricular hypertrophy in children and adolescents with essential hypertension. Circulation. 1998;97(19):1907-1911.

13. Gidding SS. Clinical and epidemiological significance of left ventricular mass assessed in children and adolescents. Circulation. 1998;97(19):1893-1894.

14. Cuspidi C, Rescaldani M, Sala C, Grassi G. Left-ventricular hypertrophy and obesity: a systematic review and meta-analysis of echocardiographic studies. J Hypertens. 2014;32(1):16-25.

15. Kinik ST, Varan B, Yildirim SV, Tokel K. The effect of obesity on echocardiographic and metabolic parameters in childhood. J Pediatr Endocrinol Metab. 2006;19(8):1007-1014.

16. Movahed MR, Bates S, Strootman D, Sattur S. Obesity in adolescence is associated with left ventricular hypertrophy and hypertension. Echocardiography. 2011;28(2):150-153.

17. Senatorova AS, Chaichenko TV, Boichenko AD. [Myocardial geometry and function in overweight and obese adolescents]. Lik Sprava. 2011;(7-8):43-51.

18. de Simone G, Devereux RB, Daniels SR, Koren MJ, Meyer RA, Laragh JH. Effect of growth on variability of left ventricular mass: assessment of allometric signals in adults and children and their capacity to predict cardiovascular risk. J Am Coll Cardiol. 1995;25(5):1056-1062.

19. Rosa EC, Moyses VA, Sesso RC, Plavnik FL, Ribeiro FF, Kohlmann NE, Ribeiro AB, et al. Left ventricular hypertrophy evaluation in obese hypertensive patients: effect of left ventricular mass index criteria. Arq Bras Cardiol. 2002;78(4):341-351.
20. Hashimoto I, Ichida F, Tsubata S, Hamamichi Y, Uese K, Miyazaki A, Miyawaki T. A novel method for indexing echocardiographic left ventricular mass in infants, children and adolescents: evaluation of obesity-induced left ventricular hypertrophy. Pediatr Int. 1999;41(2):126131.

21. Di Bonito P, Capaldo B, Forziato C, Sanguigno E, Di Fraia T, Scilla C, Cavuto L, et al. Central adiposity and left ventricular mass in obese children. Nutr Metab Cardiovasc Dis. 2008;18(9):613-617.

22. Crowley DI, Khoury PR, Urbina EM, Ippisch HM, Kimball TR. Cardiovascular impact of the pediatric obesity epidemic: higher left ventricular mass is related to higher body mass index. J Pediatr. 2011;158(5):709-714 e701.

23. Bostanci BK, Civilibal M, Elevli M, Duru NS. Ambulatory blood pressure monitoring and cardiac hypertrophy in children with metabolic syndrome. Pediatr Nephrol. 2012;27(10):1929-1935.

24. Hanevold C, Waller J, Daniels S, Portman R, Sorof J, International Pediatric Hypertension A. The effects of obesity, gender, and ethnic group on left ventricular hypertrophy and geometry in hypertensive children: a collaborative study of the International Pediatric Hypertension Association. Pediatrics. 2004;113(2):328-333.

25. Paschoal MA, Campos JF, De Moraes FM. Perfil antropometrico e clinico de escolares e sua relacao com a sindrome metabolica na infancia. Rev Bras Prom Saude. 2012;25:202-208.

26. Iampolsky MN, De Souza FIS, Sarni RO. Influencia do indice de massa corporal e da circunferencia abdominal na pressao arterial sistemica de criancas. Rev Paul Pediatr. 2010;28(2):181-187.

27. de Moraes LI, Nicola TC, de Jesus JS, Alves ER, Giovaninni NP, Marcato DG, Sampaio JD, et al. High blood pressure in children and its correlation with three definitions of obesity in childhood. Arq Bras Cardiol. 2014;102(2):175-180.

28. Falkner B, DeLoach S, Keith SW, Gidding SS. High risk blood pressure and obesity increase the risk for left ventricular hypertrophy in African-American adolescents. J Pediatr. 2013;162(1):94-100.

29. De Almeida CAN, Pinho AP, Ricco RG, Elias CP. Circunferencia abdominal como indicador de parametros clinicos e laboratoriais ligados a obesidade infantojuvenil: comparacao entre duas referencias. J Pediatr. 2007;83:181-185.

30. Taylor RW, Jones IE, Williams SM, Goulding A. Evaluation of waist circumference, waist-to-hip ratio, and the conicity index as screening tools for high trunk fat mass, as measured by dual-energy X-ray absorptiometry, in children aged 3-19 y. Am J Clin Nutr. 2000;72(2):490495. 